\title{
Update on Solar and Atmospheric Four-Neutrino Oscillations
}

\section{C. Gonzalez-Garcia*}

Theory Division, CERN, CH-1211 Geneva 23, Switzerland,

Instituto de Física Corpuscular, Universitat de València-C.S.I.C.

Edificio Institutos de Paterna, Apt 22085, 46071 València, Spain,

and C.N. Yang Institute for Theoretical Physics

State University of New York at Stony Brook Stony Brook, NY 11794-3840, USA

concepcion. gonzalez-garcia@cern.ch

\section{Maltoni and C. Peña-Garay}

Instituto de Física Corpuscular, Universitat de València-C.S.I.C.

Edificio Institutos de Paterna, Apt 22085, 46071 València, Spain

ABSTRACT: In this talk we present the update of our combined analysis of the neutrino oscillation solutions of both the solar and the atmospheric neutrino problem, in the framework of four-neutrino mixing [i]1].1.

We include in our analysis the most recent solar neutrino rates of Homestake, SAGE, GALLEX and GNO [2:2], as well as the recent 1258-day Super-Kamiokande data sample [i3i], including the recoil electron energy spectrum for both day and night periods and the recent results from the CC even rates at SNO [i⿱亠䒑口心 $]$. As for atmospheric neutrinos we include in our analysis all the contained events from the latest 79.5 kton-yr Super-Kamiokande data set as well as the upward-going neutrino-induced muon fluxes from both Super-Kamiokande

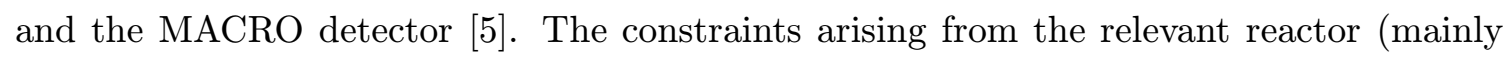

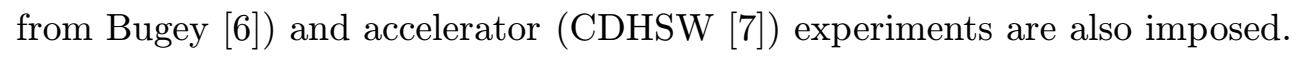

One of the most important issues in the context of 4-neutrino scenarios is the question of the 4-neutrino mass spectrum. There are six possible four-neutrino schemes that can accommodate the results from solar and atmospheric neutrino experiments as well as the LSND evidence. They can be divided in two classes: $(3+1)$ and $(2+2)$ In the $(3+1)$ schemes there is a group of three neutrino masses separated from an isolated one by a gap of the order of $1 \mathrm{eV}^{2}$, which is responsible for the short-baseline oscillations observed in the LSND experiment. In $(2+2)$ schemes there are two pairs of close masses separated by the LSND gap. The main difference between these two classes is that, if a $(2+2)$-spectrum

\footnotetext{
${ }^{*}$ Speaker.
} 
is realized in nature, the transition into the sterile neutrino is a solution of either the solar or the atmospheric neutrino problem, or the sterile neutrino has to take part in both, whereas with a $(3+1)$-spectrum it could be only slightly mixed with the active neutrinos and mainly provide a description of the LSND result. The $(3+1)$ schemes are disfavoured by experimental data with respect to the $(2+2)$ schemes $\left[\bar{\beta}_{1}^{1}, \bar{g}_{1}\right]$, but they are still marginally allowed [1] ${ }_{1}^{1} \overline{3}$. Therefore we concentrate on the $2+2$ schemes.

The main characteristic of $(2+2)$-spectra is that either solar or atmospheric oscillations must involve the sterile neutrino. Oscillation into sterile neutrinos are disfavoured for both atmospheric and solar neutrinos [2]. One expect then that these schemes will be also disfavoured. However, as first discussed by [1] $\left.{ }_{1}^{1} \overline{0}\right]$, when considered in the framework of $(2+2)$ schemes, oscillations into pure active or pure sterile states are only limiting cases of the most general possibility of oscillations into an admixture of active and sterile neutrinos. One can wonder then, if some finite admixture of active-sterile oscillations is able to give a suitable description of both solar [i, $\left.\overline{1}_{1}\right]$ and atmospheric data [i] $\left.\overline{1}_{2} \bar{i}\right]$.

For the phenomenology of neutrino oscillations in $(2+2)$ schemes the mass spectrum can be taken as: $\Delta m_{\odot}^{2}=\Delta m_{21}^{2} \ll$ $\Delta m_{\mathrm{atm}}^{2}=\Delta m_{43}^{2} \ll \Delta m_{\mathrm{LSND}}^{2}=\Delta m_{41}^{2} \simeq$ $\Delta m_{42}^{2} \simeq \Delta m_{31}^{2} \simeq \Delta m_{32}^{2}$ and neglecting possible CP phases, the matrix $U$ can be written as a product of six rotations, $U_{12}, U_{13}$, $U_{14}, U_{23}, U_{24}$ and $U_{34}$ where $U_{i j}$ represents a rotation of angle $\theta_{i j}$ in the $i j$ plane. The order of the product of the matrices corresponds to a specific parametrization of the mixing matrix $U$ and in order to study oscillations of the solar and atmospheric neutri-

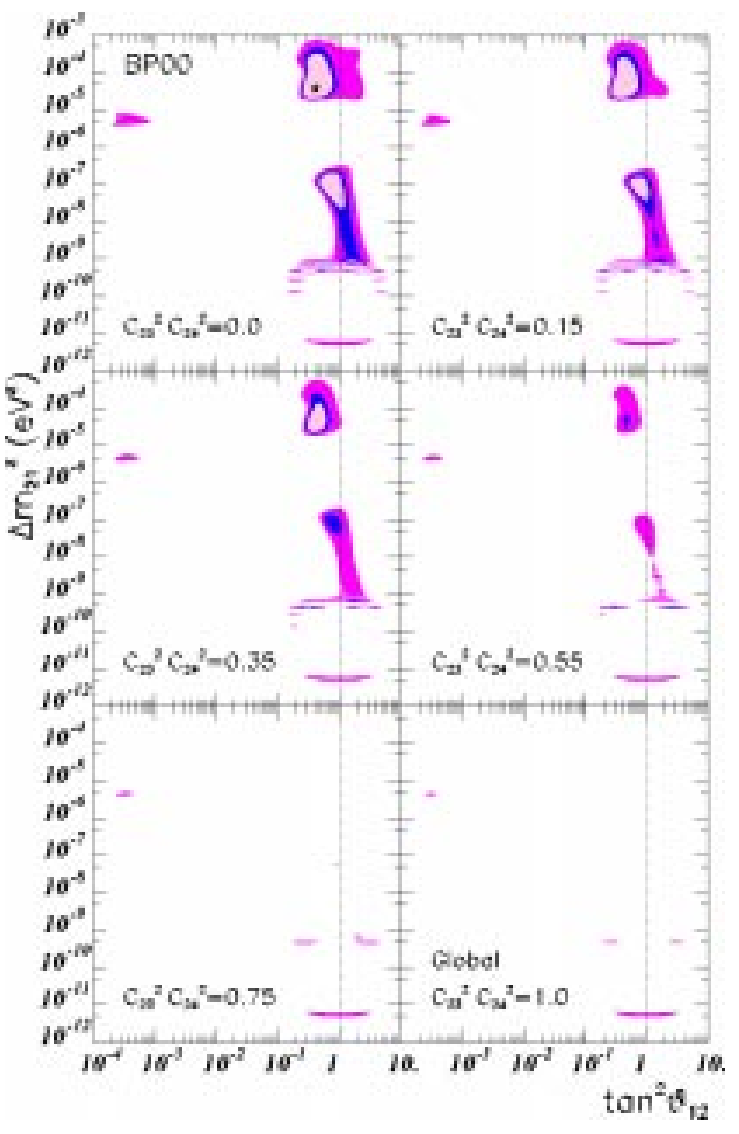

Figure 1: Results of the global analysis of solar neutrino data for the allowed regions in $\Delta m_{21}^{2}$ and $\tan ^{2} \theta_{12}$ for the $2+2$ four-neutrino oscillations. The different panels represent sections at a given value of the active-sterile admixture $\left|U_{s 1}\right|^{2}+\left|U_{s 2}\right|^{2}=c_{23}^{2} c_{24}^{2}$ of the three-dimensional allowed regions at $90 \%, 95 \%$ and $99 \% \mathrm{CL}$. The best-fit point in the three-parameter space is plotted as a star. nos, which include the matter effects in the Sun and/or in the Earth, it is convenient to use the parametrization: $U=U_{24} U_{23} U_{14} U_{13} U_{34} U_{12}$. This general form can be further simplified by taking into account the negative results from the reactor experiments, in particular the Bugey experiment, which in the range of $\Delta m_{41}^{2}$ relevant to the LSND experiment implies that $\left|U_{e 3}\right|^{2}+\left|U_{e 4}\right|^{2}=c_{14}^{2} s_{13}^{2}+s_{14}^{2} \lesssim 10^{-2}$ so that the two angles $\theta_{13}$ and $\theta_{14}$ give negligible contributions to solar and atmospheric neutrino 
transitions for which the $U$ matrix takes the effective form:

$$
U=\left(\begin{array}{cccc}
c_{12} & s_{12} & 0 & 0 \\
-s_{12} c_{23} c_{24} & c_{12} c_{23} c_{24} & s_{23} c_{24} c_{34}-s_{24} s_{34} & s_{23} c_{24} s_{34}+s_{24} c_{34} \\
s_{12} s_{23} & -c_{12} s_{23} & c_{23} c_{34} & c_{23} s_{34} \\
s_{12} c_{23} s_{24} & -c_{12} c_{23} s_{24} & -s_{23} s_{24} c_{34}-c_{24} s_{34} & -s_{23} s_{24} s_{34}+c_{24} c_{34}
\end{array}\right) .
$$

Concerning the range of variation of the mixing angles, the full parameter space relevant to solar and atmospheric neutrino oscillation can be covered by choosing the mixing angles in the ranges: $0 \leq \theta_{12} \leq \frac{\pi}{2}, 0 \leq \theta_{23} \leq \frac{\pi}{2}, 0 \leq \theta_{24} \leq \frac{\pi}{2}$, and $-\frac{\pi}{2} \leq \theta_{34} \leq \frac{\pi}{2}$.

In this scheme solar neutrino oscillations are generated by the mass-square difference between $\nu_{2}$ and $\nu_{1}$ while atmospheric neutrino oscillations are generated by the mass-squared difference between $\nu_{3}$ and $\nu_{4}$. It is clear from Eq. (iiil) that the survival of solar $\nu_{e}$ 's mainly depends on the mixing angle $\theta_{12}$ while atmospheric $\nu_{e}$ 's are not affected by the four-neutrino oscillations in the approximation $\theta_{13}=\theta_{14}=0$ and neglecting the effect of $\Delta m_{21}^{2}$ in the range of atmospheric neutrino energies. Con-

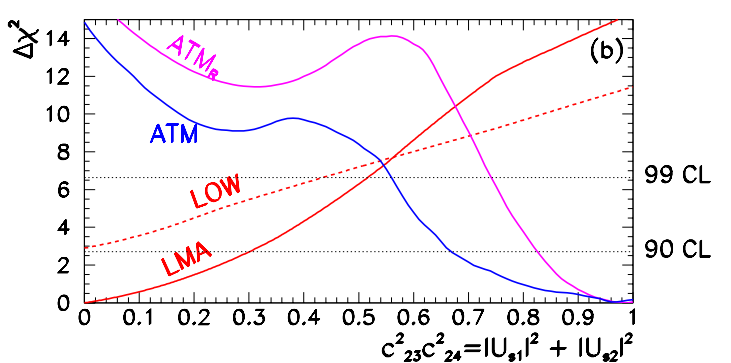

Figure 2: $\Delta \chi^{2}$ as a function of the active-sterile admixture $\left|U_{s 1}\right|^{2}+\left|U_{s 2}\right|^{2}=c_{23}^{2} c_{24}^{2}$ for the analysis of solar and atmospheric data in $2+2$ four-neutrino schemes. versely, the survival probability of atmospheric $\nu_{\mu}$ 's mainly depends on the mixing angle $\theta_{34}$. Thus solar neutrino oscillations occur between the states $\nu_{e} \rightarrow \nu_{\alpha}$ with $\nu_{\alpha}=$ $c_{23} c_{24} \nu_{s}+\sqrt{1-c_{23}^{2} c_{24}^{2}} \nu_{a}$ with mixing angle $\theta_{12} . \nu_{a}$ is a linear combination of $\nu_{\mu}$ and $\nu_{\tau}$. Let us remind the reader that $\nu_{\mu}$ and $\nu_{\tau}$ cannot be distinguished in solar neutrino experiments, because their matter potential and their interaction in the detectors are equal, due only to $\mathrm{NC}$ weak interactions. Thus solar neutrino oscillations cannot depend on the mixing angle $\theta_{34}$ and depend on $\theta_{23}$ and $\theta_{24}$ through the combination $c_{23}^{2} c_{24}^{2}$.

Atmospheric neutrino oscillations, i.e. oscillations with the mass difference $\Delta m_{34}^{2}$ and mixing angle $\theta_{34}$, occur between the states

$$
\nu_{\beta} \rightarrow \nu_{\gamma} \quad \text { with } \quad \nu_{\beta}=s_{23} c_{24} \nu_{s}+c_{23} \nu_{\mu}-s_{23} s_{24} \nu_{\tau} \quad \text { and } \quad \nu_{\gamma}=s_{24} \nu_{s}+c_{24} \nu_{\tau} .
$$

From the previous discussion we see that the mixing angles $\theta_{23}$ and $\theta_{24}$ determine two projections corresponding to $c_{23}^{2} c_{24}^{2}=1-\left|U_{a 1}\right|^{2}-\left|U_{a 2}\right|^{2}=\left|U_{s 1}\right|^{2}+\left|U_{s 2}\right|^{2}$ which gives the size of the projection of the sterile neutrino onto the state in which the solar $\nu_{e}$ oscillates, and $s_{23}^{2}=\left|U_{\mu 1}\right|^{2}+\left|U_{\mu 2}\right|^{2}=1-\left|U_{\mu 3}\right|^{2}-\left|U_{\mu 4}\right|^{2} \lesssim 0.2$ which determines the size of the projection of the $\nu_{\mu}$ over the "atmospheric" neutrino oscillating states. One expects $s_{23}$ to be small in order to explain the atmospheric neutrino deficit and, as we will see this is the case. Furthermore, the negative results from the CDHS and CCFR experiments on searches for $\nu_{\mu}$-disappearance also constrain such a projection to be smaller than 0.2 at $90 \% \mathrm{CL}$ for $\Delta m_{\mathrm{LSND}}^{2} \gtrsim 0.4 \mathrm{eV}^{2}$. We distinguish the following limiting cases:

- if $c_{23}=1$ then $U_{\mu 1}=U_{\mu 2}=0$. The atmospheric $\nu_{\mu}=\nu_{\beta}$ state oscillates into a state $\nu_{\gamma}=c_{24} \nu_{\tau}+s_{24} \nu_{s}$. We will denote this case as "restricted". In particular: 
- in the case $c_{23}=c_{24}=1, U_{a 1}=U_{a 2}=0\left(U_{s 3}=U_{s 4}=0\right)$ and we have the limit of pure two-generation solar $\nu_{e} \rightarrow \nu_{s}$ transitions and atmospheric $\nu_{\mu} \rightarrow \nu_{\tau}$ transitions

- in the case $c_{24}=0$ then $U_{s 1}=U_{s 2}=0$ and $U_{\tau 3}=U_{\tau 4}=0$, corresponding to the limit of pure two-generation solar $\nu_{e} \rightarrow \nu_{\tau}$ transitions and atmospheric $\nu_{\mu} \rightarrow \nu_{s}$ transitions.

- if $c_{23}=0, U_{s 1}=U_{s 2}=0$ corresponding to the limit of pure two-generation solar $\nu_{e} \rightarrow \nu_{a}$ with $a=\mu$ and there are no atmospheric neutrino oscillations as the projection of $\nu_{\mu}$ over the relevant states cancels out $\left(U_{\mu 3}=U_{\mu 4}=0\right)$.

Notice that in the limiting case $\theta_{23}=$ 0 , there is an additional symmetry in the relevant probabilities so that the full parameter space can be spanned by $0 \leq \theta_{34} \leq$ $\frac{\pi}{2}$, as expected since in this case we have the effective two-neutrino oscillations solar $\nu_{e} \rightarrow \nu_{\alpha}$ and atmospheric $\nu_{\mu} \rightarrow \nu_{\gamma}$.

With all this we find that the survival probabilities for solar neutrinos take the form

$$
\begin{aligned}
& P_{\nu_{e} \rightarrow \nu_{s}}^{\text {Sun }+ \text { Earth }}=c_{23}^{2} c_{24}^{2}\left(1-P_{\nu_{e} \rightarrow \nu_{e}}^{\text {Sun }+ \text { Earth }}\right) \\
& P_{\nu_{e} \rightarrow \nu_{a}}^{\text {Sunth }}=\left(1-c_{23}^{2} c_{24}^{2}\right)\left(1-P_{\nu_{e} \rightarrow \nu_{e}}^{\text {Sun Earth }}\right)
\end{aligned}
$$

where $P_{\nu_{e} \rightarrow \nu_{e}}^{S u n+E a r t h}$ takes the standard twoneutrino oscillation form for $\Delta m_{21}^{2}$ and $\theta_{12}$, found by numerically solving the evolution equation in the Sun and the Earth matter with the modified matter potential $A \equiv A_{C C}+c_{23}^{2} c_{24}^{2} A_{N C}$ with $A_{C C}=$ $2 \sqrt{2} G_{F} E N_{e}$ and $A_{N C}=-\sqrt{2} G_{F} E N_{n}$.

For atmospheric $\nu_{\mu}$, the survival probability including matter effects in the Earth can be calculated by numerically integrating the evolution equation in the Earth with a modified matter potential including the effect of the mixing angles $\theta_{23}$ and

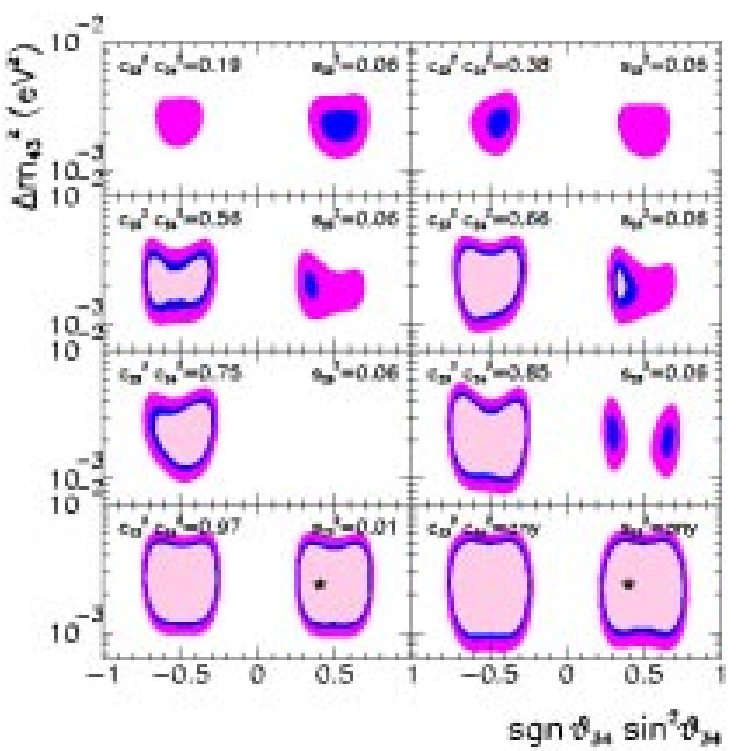

Figure 3: Results of the analysis of atmospheric neutrino data for the allowed regions in $\Delta m_{43}^{2}$ and $\theta_{34}$ for the $2+2$ four-neutrino oscillations. The different panels represent sections at given values of the $\nu_{\mu}$ projection $\left|U_{\mu 1}\right|^{2}+\left|U_{\mu 2}\right|^{2}=s_{23}^{2}$ and the active-sterile admixture $\left|U_{s 1}\right|^{2}+\left|U_{s 2}\right|^{2}=c_{23}^{2} c_{24}^{2}$ of the four-dimensional allowed regions at $90 \%$, $95 \%$ and $99 \%$ CL. The best-fit point in the fourparameter space is plotted as a star. The last panel corresponds to the case in which $\chi^{2}$ has also been minimized with respect to $s_{23}^{2}$ and $c_{23}^{2} c_{24}^{2}$. $\theta_{24}$. For example the diagonal piece of the potential takes the form $A \equiv\left(s_{24}^{2}-s_{23}^{2} c_{24}^{2}\right) A_{N C}$ so that for pure atmospheric $\nu_{\mu} \rightarrow \nu_{\tau}$ oscillations $\left(s_{23}^{2}=s_{24}^{2}=0\right) A=0$, while for $\nu_{\mu} \rightarrow \nu_{s}\left(s_{23}^{2}=0, s_{24}^{2}=1\right) A=A_{N C}$, as expected. It is this modification of the Earth matter potential that gives the atmospheric neutrino data the capability to discriminate between the active and sterile oscillation solution. In particular, higher sensitivity to this potential effect is achievable for the higher energy part of the atmospheric neutrino flux, which lead to the upward going muon data. The main effect of the presence of this potential is that for pure active oscillations the angular distribution of upgoing muons is expected to be steeper at larger arrival angles, while a flattening is expected from the matter effects for sterile oscillations. 
Summarizing solar neutrino oscillations depend only on the new mixing angles through the product $c_{23} c_{24}$ and therefore the analysis of the solar neutrino data in four-neutrino mixing schemes is equivalent to the two-neutrino analysis but taking into account that the parameter space is now three-dimensional $\left(\Delta m_{21}^{2}, \tan ^{2} \theta_{12}, c_{23}^{2} c_{24}^{2}\right)$. Although originally this derivation was performed in the framework of the $(2+2)$ schemes, it is equally valid for the $(3+1)$ ones. On the contrary, the angles $\theta_{23}$ and $\theta_{24}$ enter independently in the atmospheric oscillations and the analysis of the the analysis of the atmospheric neutrino data in the four-neutrino mixing schemes is equivalent to the two-neutrino analysis, but taking into account that the parameter space is now four-dimensional $\left(\Delta m_{43}^{2}, \theta_{34}, c_{23}^{2}, c_{24}^{2}\right)$.

In Fig. $\underline{I}_{1}^{\prime}$ we show the allowed regions for the oscillation parameters $\Delta m_{21}$ and $\tan ^{2}\left(\theta_{12}\right)$ from the global analysis of the solar neutrino data in the framework of four-neutrino oscillations for different values of $c_{23}^{2} c_{24}^{2}$. The global minimum used in the construction of the regions lies in the LMA region and for pure $\nu_{e}$-active oscillations. As seen in the figure as the sterile component increases the regions became smaller till they totally disappear.

This behaviour is also illustrated in Fig. 12i-1 where we show the shift on the $\chi^{2}$ for the analysis of the solar data in the framework of oscillations between four neutrinos as a function of the active-sterile admixture $\left|U_{s 1}\right|^{2}+\left|U_{s 2}\right|^{2}=c_{23}^{2} c_{24}^{2}$. From the figure we find that:

- solar neutrino data favour pure $\nu_{e} \rightarrow \nu_{a}$ oscillations but sizeable active-sterile admixtures are still allowed.

- the three-dimensional regions are acceptable at 90\% (99\%) CL for

$$
\begin{array}{ll}
\left|U_{s 1}\right|^{2}+\left|U_{s 2}\right|^{2}=c_{23}^{2} c_{24}^{2}<0.45 \quad(0.72) & \text { for LMA, } \\
\left|U_{s 1}\right|^{2}+\left|U_{s 2}\right|^{2}=c_{23}^{2} c_{24}^{2}<0.40 \quad(0.99) & \text { for LOW-QVO; }
\end{array}
$$

- at $99 \%$ CL both regions are allowed for maximal active-sterile mixing $c_{23}^{2} c_{24}^{2}=0.5$.

For the analysis of the atmospheric data in Fig. $\bar{\beta}_{1}^{1}$ we plot the sections of such a volume in the plane for different values of the projections $\left|U_{\mu 1}\right|^{2}+\left|U_{\mu 2}\right|^{2}$ and $\left|U_{s 1}\right|^{2}+\left|U_{s 2}\right|^{2}$. The global minimum used in the construction of the regions lies almost in the pure atmospheric $\nu_{\mu}-\nu_{\tau}$ oscillations. As shown in the figure the regions becomes considerably smaller for increasing values of the mixing angle $\theta_{23}$, which determines the size of the projection of the $\nu_{\mu}$ over the "atmospheric" neutrino oscillating states, and for increasing values of the mixing angle $\theta_{24}$, which determines the active-sterile admixture in which the "almost- $\nu_{\mu}$ " oscillates. Therefore from the analysis of the atmospheric neutrino data we obtain an upper bound on both mixings, which, in particular, implies a lower bound on the combination $c_{23}^{2} c_{24}^{2}=\left|U_{s 1}\right|^{2}+\left|U_{s 2}\right|^{2}$ limited from above by the solar neutrino data. Fig. $\underline{2}_{1}^{\overline{1}}$ we show the shift on the $\chi^{2}$ for the analysis of the atmospheric data in the framework of oscillations between four neutrinos as a function of the active-sterile admixture $\left|U_{s 1}\right|^{2}+\left|U_{s 2}\right|^{2}=c_{23}^{2} c_{24}^{2}$ for the general case (in which the analysis is optimized with respect to the parameter $\left.s_{23}^{2}=\left|U_{\mu 1}\right|^{2}+\left|U_{\mu 2}\right|^{2}\right)$ as well as the restricted case in which $s_{23}^{2}=0$. The $90 \%$ (99\%) CL lower bounds on $c_{23}^{2} c_{24}^{2}$ from the analysis of the atmospheric neutrino data are:

$$
\begin{aligned}
c_{23}^{2} c_{24}^{2} & =\left|U_{s 1}\right|^{2}+\left|U_{s 2}\right|^{2}>0.64(0.52) \\
c_{24}^{2} & =\left|U_{s 1}\right|^{2}+\left|U_{s 2}\right|^{2}>0.83(0.74) \quad \text { for the restricted case. }
\end{aligned}
$$


In summary the analysis of the solar data favours the scenario in which the solar oscillations in the plane 1-2 are $\nu_{e}$ oscillations into an active neutrino, and from that analysis one finds an upper limit on the projection of the $\nu_{s}$ on the 1-2 states. On the other hand, the atmospheric neutrino analysis prefers the oscillations of the 3-4 states to occur between a close-to-pure $\nu_{\mu}$ and an active $\left(\nu_{\tau}\right)$ neutrino, thus giving an upper bound on the projection of the $\nu_{s}$ over the 3-4 states, or equivalently a lower bound on its projection over the 1-2 states. The open question is then what the best scenario is for the active-sterile admixture once these two bounds are put together. The result from the combined analysis still favours close-to-pure active and sterile oscillations (what gives either a bad description to solar or to atmospheric data) and disfavours oscillations into a near-maximal active-sterile admixture which could, in principle, lead to a compromise in the description of both data samples. This can be interpreted as a hint of a incompatibility between the present solar, atmospheric and LSND results even in the context of $2+2$ four-neutrino mixing although, at present, these schemes cannot be ruled out from the pure statistical point of view.

The work is supported by the European Union Marie-Curie fellowships HPMF-CT2000-01008 and HPMF-CT-2000-00516, by grants PB98-0693, PB97-1261, GV99-3-1-01, by RTN network HPRN-CT-2000-00148 and by the ESF network grant N. 86 .

\section{References}

[1] M. C. Gonzalez-Garcia, M. Maltoni and C. Pena-Garay, Phys. Rev. D 64, 093001 (2001)

[2] See plenary talk by K. Nishikawa in these proceedings,

[3] See Super-Kamiokande presentation by S. Moriyama in these proceedings,

[4] See talk by A. MacDonald in these proceedings.

[5] See talk E.Scapparone by in these proceedings,

[6] B. Achkar et al., Nucl. Phys. B424, 503 (1995).

[7] F. Dydak et al., Phys. Lett. B 134, 281 (1984).

[8] S.M. Bilenky et al., Eur. Phys. J. C 1, 247 (1998); Phys. Rev. D60, 073007 (1999).

[9] V. Barger, S. Pakvasa, T. J. Weiler, and K. Whisnant, Phys. Rev. D58, 093016 (1998).

[10] D. Dooling, C. Giunti, K. Kang and C.W. Kim, Phys. Rev. D 61, 073011 (2000).

[11] C. Giunti, M. C. Gonzalez-Garcia and C. Peña-Garay, Phys. Rev. D62, 013005 (2000).

[12] G.L. Fogli et al., Phys. Rev. D 63, 053008 (2001); O. Yasuda, hep-ph/0006319.

[13] V. Barger et al., Phys. Lett. B489, 345 (2000); O. L. G. Peres and A. Yu. Smirnov, Nucl. Phys. B 599, 3 (2001); W. Grimus and T. Schwetz, Eur. Phys. J. C 20, 1 (2001) 